\title{
Volterra analysis of the Non-linear Magneto-Optic Recording Channel
}

\author{
C. Tannous and J. Gieraltowski \\ Laboratoire de Magnétisme de Bretagne, UMR CNRS 6135 , \\ Université de Bretagne Occidentale, BP: 809 Brest CEDEX, 29285 FRANCE
}

\begin{abstract}
The non-linearities arising in the magneto-optical recording write channel are described. Their occurrence result in distortions that are damaging to the information circulating across the channel. For high speed data recording it is imperative to have a precise view of the transmission characteristics of a signal sent across the channel. When the non-linearity is weak, it is possible to perform Volterra series expansions to model the time response and port it to the frequency domain where non-linear distortions are derived from basic principles and applied to a general time dependent recording write channel.
\end{abstract}

Key words: Non-linear channel. Non-linear distortions. Magneto-optical Recording.

\section{Introduction}

Harmonic and Intermodulation distortions occur when a signal at a single frequency or a superposition of several signals with different frequencies propagate in a non-linear physical system. Historically it was first highlighted in the Radar and Radio Communications industry because of interest in understanding interference from other radars, jammers, other transmitters or modulators... Presently, it is pervading High Energy Physics particle detectors, wideband amplifiers, satellite communications [1] and other areas of science and technology. To cite some of the areas of interest, it occurs in many types of devices such as Mechanical [2], Acoustical (Microphones for instance), Electronic [3] and Microelectronic [4], Microwave [5], Optical [6], Magnetic [7] and Superconducting [8].

Parasitic frequency terms appear either at integer multiple of the base frequency (harmonic) or as a mixture of two or several multiples of base frequencies (intermodulation) when several base frequencies are used (as in modulation systems for instance). These terms can be either postfiltered or the signal can be pre-distorted in order to avoid the appearance of these unwanted terms.

The Magneto-Optic (MO) Write channel is non-linear for several reasons. In the Laser power modulation process (LM) used for high data rates, non-linearities occur between the output power versus input current. We call this the laser dynamics level (LD). Other non-linearities can occur at the level of the laser beam magnetic layer interaction (we call this the Laser-Material level or LM level) and finally in the magnetic layer itself in the magnetodynamic process (magnetization reversal). We call this final step in the thermomagnetic write process, the MD level.

In this work, we introduce a general and systematic method to evaluate harmonic and intermodulation distortions terms of any arbitrary order directly from the equations of motion describing the physical system at hand. The distortions terms are evaluated with the time domain Volterra expansion transformed into the frequency domain.

This work is organised as follows: In section 2, the origin of the different non-linear terms of the MO channel are described. In section 3, the Volterra expansion is performed in the time domain, then Fourier transformed to yield the distortion terms in the frequency domain. Section 4 bears an illustration of a full Volterra expansion of a simple example with the complete evaluation of the distortions and finally section 5 contains the conclusions.

\section{Description of the non-linear MO channel}

The physical processes in the MO Recording Write channel span at least three levels of interest. In the LIMDOW (Light Intensity Modulated Direct Overwrite) process a polarised laser light source is driven by a modulating injection current. Larger values of the current obtained from modulation or through increase of signal to noise ratio might induce undesired non-linearities in the laser output power creating undesired frequency components in the LIMDOW process.

The polarisation and the laser mode determine the shape and the width of the laser hotspot. For instance, the Gaussian laser beam with the lowest divergence angle is provided by the $T E M_{00}$ mode, whereas the $T E M_{01}^{*}$ mode (given by the superposition of the $T E M_{01}$ and $T E M_{10}$ modes ) is doughnut shaped favoring radial rather than axial heat conduction. The actual temperature distribution useful for the thermomagnetic write process is determined from the laser mode and the temperature dependence of the thermal conductivity of the material. The optical $\mathbf{E}$ field created by the laser, heating the material in the LIMDOW process varies rapidly from a low to a high value that might induce non-linearities in the material response. If the latter is magnetic, the resulting polarisation can be described with a power series in the optical $\mathbf{E}$ field involving higher order response functions (susceptibility tensors) depending on the polarization direction, frequency and the applied magnetic field.

Finally the non-linearity of the magnetic response arises from the equation of motion describing magnetization evolution driven by the thermomagnetic write operation. Several forces must be considered: those acting on the bubblelike domain formed with the thermomagnetic process, the coercive force and the force due to the wall energy gra- 
dient in the temperature profile induced by the laser spot. Phenomenologically, it can be said that all these forces act on the magnetization to alter its state and reverse it. For simplicity, we consider that magnetization $\mathbf{M}$ dynamics is rotational and obeys the Landau-Lifshitz-Gilbert (L-L-G) equation:

$$
\frac{d \mathbf{M}}{d t}=\gamma\left(\mathbf{M} \times \mathbf{H}_{e f f}\right)-\alpha \gamma \frac{\mathbf{M} \times\left(\mathbf{M} \times \mathbf{H}_{e f f}\right)}{|\mathbf{M}|}
$$

where $\mathbf{H}_{e f f}$ the effective field is given by:

$$
\mathbf{H}_{e f f}=-\frac{\partial U}{\partial \mathbf{M}}
$$

where $U$ is the total energy of the bubble-like domain, $\gamma$ the gyromagnetic ratio and $\alpha$ the damping parameter.

The usual first order frequency permeability response $\mu(\omega)$ is generally calculated directly from the L-L-G equation or after a linearisation procedure $[9,10]$. However in our case we can either expand the response $\mathbf{M}$ versus the field $\mathbf{H}_{e f f}$ to a higher order similarly to the Electric case or perform the direct integration of the L-L-G equation with Fast Fourier techniques [9]. The different procedures depend on whether a time-dependent or frequency dependent methodology is used. In the next section, we develop a frequency based methodology in order to decribe the channel nonlinearities.

\section{Volterra description of the MO Write Channel}

A non-linear time-dependent communication channel described as a scalar input-output relationship might be viewed as a characteristic $i(t)=F[v(t)]$ with input $v(t)$ and output $i(t)$ where $F$ is a non-linear functional that depends in general on $v(t)$ as well as its higher order time derivatives $v^{\prime}(t), v^{\prime \prime}(t) \ldots$ In the simple case, when $F[v(t)]$ depends only on $v(t)$, the Volterra expansion entails expressing the response $i(t)$ versus $v(t)$ in the form of a series expansion with the help of generalised impulse responses $h_{n}$ in the following way:

$$
\begin{array}{r}
i(t)=\int_{0}^{\infty} h_{1}\left(t_{1} ; t\right) v\left(t_{1}\right) d t_{1} \\
+\int_{0}^{\infty} \int_{0}^{\infty} h_{2}\left(t_{1}, t_{2} ; t\right) v\left(t_{1}\right) v\left(t_{2}\right) d t_{1} d t_{2} \\
+\int_{0}^{\infty} \int_{0}^{\infty} \int_{0}^{\infty} h_{3}\left(t_{1}, t_{2}, t_{3} ; t\right) v\left(t_{1}\right) v\left(t_{2}\right) v\left(t_{3}\right) d t_{1} d t_{2} d t_{3} \ldots
\end{array}
$$

The Fourier transforms of the impulse response functions $h_{n}$ are the generalised $\mathrm{n}^{\text {th }}$ order transfer functions $H_{n}\left(\omega_{1}, \omega_{2}, \omega_{3} \ldots\right)$ :

$$
\begin{array}{r}
H_{n}\left(j \omega_{1}, j \omega_{2}, j \omega_{3} \ldots\right)=\int_{0}^{\infty} \int_{0}^{\infty} \ldots \int_{0}^{\infty} h_{n}\left(t_{1}, t_{2}, t_{3}, \ldots\right) \\
\times e^{-j\left(\omega_{1} t_{1}+\omega_{2} t_{2}+\omega_{3} t_{3} \ldots\right)} d t_{1} d t_{2} d t_{3} \ldots
\end{array}
$$

When the input-output relationship is vectorial with the form $\mathbf{P}(t)=\mathbf{F}[\mathbf{E}(t)]$ where $\mathbf{F}$ is a vector functional relating the input electrical field $\mathbf{E}$ to the output material polarization $\mathbf{P}$, the generalised impulse responses $h_{n}$ acquire a tensor character. After taking the Fourier transform one obtains the material polarization $\mathbf{P}$ expansion in terms of the E field as:

$$
\begin{aligned}
& \mathbf{P}(t)=\int_{0}^{\infty} \chi_{1}\left(t_{1} ; t\right) \mathbf{E}\left(t_{1}\right) d t_{1} \\
& +\int_{0}^{\infty} \int_{0}^{\infty} \chi_{2}\left(t_{1}, t_{2} ; t\right) \mathbf{E}\left(t_{1}\right) \mathbf{E}\left(t_{2}\right) d t_{1} d t_{2} \\
& +\int_{0}^{\infty} \int_{0}^{\infty} \int_{0}^{\infty} \chi_{3}\left(t_{1}, t_{2}, t_{3} ; t\right) \\
& \quad \times \mathbf{E}\left(t_{1}\right) \mathbf{E}\left(t_{2}\right) \mathbf{E}\left(t_{3}\right) d t_{1} d t_{2} d t_{3} \ldots
\end{aligned}
$$

where the susceptibility tensors $\chi_{1,2,3}$ are the tensor counterparts of the scalar impulse response functions $h_{n}$. In general, they depend on the polarization directions and the applied magnetic field. The familiar expression below is simply the Fourier transform of the above expression:

$$
\hat{\mathbf{P}}=\hat{\chi_{1}} \hat{\mathbf{E}}+\hat{\chi_{2}} \hat{\mathbf{E}} \hat{\mathbf{E}}+\hat{\chi_{3}} \hat{\mathbf{E}} \hat{\mathbf{E}} \hat{\mathbf{E}} \ldots
$$

where $\hat{\mathbf{P}}, \hat{\mathbf{E}}$ and $\hat{\chi}_{1,2,3}$ are the Fourier transform of the corresponding quatities.

The $\mathrm{n}^{\text {th }}$ order non-linear harmonic and intermodulation distortions are calculated from the generalised $\mathrm{n}^{t h}$ order transfer functions $H_{n}\left(\omega_{1}, \omega_{2}, \omega_{3} \ldots\right)$. For instance, the n-th order distortion is given by:

$$
\begin{aligned}
& M_{n}\left( \pm j \omega_{1}, \pm j \omega_{2}, \pm j \omega_{3} \ldots\right)= \\
& 20 \log _{10}\left\{\frac{\left|H_{n}\left( \pm j \omega_{1}, \pm j \omega_{2}, \pm j \omega_{3} \ldots\right)\right|}{2^{n-1} \prod_{m=1}^{n}\left|H_{1}\left( \pm j \omega_{m}\right)\right|}\right\}
\end{aligned}
$$

When the non-linear functional $F$ depends on $v(t)$ and its higher order time derivatives $v^{\prime}(t), v^{\prime \prime}(t) \ldots$ the Harmonic Input Method (HIM) is used. This method allows easily the calculation of the Volterra transfer functions $H_{n}$. Express the input $v$ as the sum:

$$
v=\exp \left(j \omega_{1} t\right)+\exp \left(j \omega_{2} t\right)+\epsilon x p\left(j \omega_{3} t\right) \ldots
$$

The output $i$ is written as the sum:

$$
i=\sum_{k, l, m \ldots=0}^{\infty} G_{k l m \ldots} \exp \left[j\left(k \omega_{1}+l \omega_{2}+m \omega_{3} \ldots\right) t\right]
$$

The different $H_{n}$ 's are found by direct identification of the $G_{k l m \ldots}$ coefficients as illustrated in the next section. Calling the time-dependent input-output characteristic $i(t)=$ $F[v(t)]$ where $i, v$ and $F$ are scalar or vector quantities, we consider the MO channel at the LD level for instance. In this case, the coupled Laser rate equations possess a scalar input $i$, the injection current and a vector output given by the set of photon population number for each mode $s_{1}, s_{2}, s_{3}, \ldots s_{m}$ where $m$ is the number of modes.

When we consider the MO channel at the LM or MD level, retardation effects occurring in magnetic systems should be accounted for. In the frequency domain the latter might be treated phenomenologically as phase factors altering the generalised transfer functions $H_{n}$. Hysteresis phenomena 
in the general case (Non-linearity + Delay) transforms the non-linear evolution equations (basically, differential equations) into Delay-Differential equations like in amplifieroutput transformers or group filters. That means, the number of coupled equations becomes infinite.

The frequency range of the interaction between the magnetization and light spans a wide range. As the frequency changes, different non-linear spin-photon processes must be considered (for an early review, see reference [11]). Lim et al. [7] consider two main frequency ranges:

a- The optical frequency range, where the above Tensor susceptibilities are considered.

b- The rf range, where the L-L-G equation is inverted to yield the magnetization as a power series with respect to the exciting rf field like the polarization versus the electric field.

Parasitic frequency terms appear either at integer multiple of the base frequency (harmonic) or as a mixture of two or several multiples of base frequencies (inter-modulation) when several base frequencies are used (as in modulation systems, for instance). These terms can be either postfiltered or the signal can be pre-distorted in order to avoid the appearance of parasites at frequencies of interest.

\section{Example illustrating the Volterra Analysis}

Our approach can handle an arbitrary number of coupled dynamic evolution equations. For simplicity we apply our methodology, to the case of two coupled first-order ordinary differential equations describing photon population and electron population in a laser diode. The method is in fact valid for any number of population equations as long as the elimination procedure of all intermediate variables (or populations) is possible, leaving us with a single equation relating input to output. In this case the input is the injection current $I$ and the output is the $S$ the photon density of the single mode (SM or $m=1$ ) yielding the power produced by the laser. The coupled rate equations are written as:

$$
\begin{aligned}
\frac{d N}{d t} & =\frac{I}{q V}-\frac{N}{\tau_{n}}-g\left(N-N_{t}\right)(1-\epsilon S) S \\
\frac{d S}{d t} & =\frac{\Gamma \beta N}{\tau_{n}}-\frac{S}{\tau_{p h}}+\Gamma g\left(N-N_{t}\right)(1-\epsilon S) S
\end{aligned}
$$

$N_{t}$ is the electron density at transparency, $\tau_{n}$ is the electron spontaneous lifetime and $\tau_{p h}$ is the photon lifetime. $\beta$ is the fraction of spontaneous emission coupled into the lasing mode, $\Gamma$ the optical confinement factor, $g$ is the differential gain and $\epsilon$ is the gain compression parameter. $q$ is the electron charge, and $V$ the volume of the active region.

\subsection{Elimination of $\left(N-N_{t}\right)$}

The input is the injection current $I$ and the output is the light intensity represented by $S$. Since other variables such as $N$, the electron density, intervene in the SM equations we proceed to its elimination by replacing terms containing the sole variable $N$ in (10) and (11) by $\left(N-N_{t}\right)+N_{t}$ leaving everywhere the variable $\left(N-N_{t}\right)$. When several modes are present (or other intermediate variables) we proceed in an analogous manner by successive elimination until we have a single equation relating input to output. From equation (11) we extract the value of $\left(N-N_{t}\right)$ as:

$$
\left(N-N_{t}\right)=\left[\frac{\frac{d S}{d t}-\frac{\Gamma \beta N_{t}}{\tau_{n}}+\frac{S}{\tau_{p h}}}{\Gamma g(1-\epsilon S) S+\frac{\Gamma \beta}{\tau_{n}}}\right]
$$

This can be used to find $d N / d t$ and eliminate $N$ completely from equation (10). The result is:

$$
\begin{array}{r}
\frac{d}{d t}\left[\frac{\frac{d S}{d t}-\frac{\Gamma \beta N_{t}}{\tau_{n}}+\frac{S}{\tau_{p h}}}{\Gamma g(1-\epsilon S) S+\frac{\Gamma \beta}{\tau_{n}}}\right]=\frac{I}{q V}-\frac{N_{t}}{\tau_{n}} \\
-\left[g(1-\epsilon S) S+\frac{1}{\tau_{n}}\right]\left[\frac{\frac{d S}{d t}-\frac{\Gamma \beta N_{t}}{\tau_{n}}+\frac{S}{\tau_{p h}}}{\Gamma g(1-\epsilon S) S+\frac{\Gamma \beta}{\tau_{n}}}\right]
\end{array}
$$

\subsection{Small signal expansion about a static operating point}

The useful SM regime operates an injection current that modulates the output light intensity. In the small dynamic signal case, $I=I_{0}+i$ and $S=S_{0}+s$, where $I_{0}$ and $S_{0}$ are the static injection current and photon density respectively, whereas, $i=i(t)$ and $s=s(t)$, are the signals of interest. $i=i(t)$ and $s=s(t)$ play the role of input-output as explained above. We expand (13) [to $n$-th order]:

$$
\begin{aligned}
& i=\left\{A_{1} s+B_{1} s^{\prime}+C_{1} s^{\prime \prime}\right\}+\left\{A_{2} s^{2}+B_{2} s s^{\prime}\right. \\
& \left.+C_{2} s s^{\prime \prime}+D_{2}\left[s^{\prime}\right]^{2}\right\} \ldots+\left\{A_{n} s^{n}+B_{n} s^{n-1} s^{\prime}\right. \\
& \left.+C_{n} s^{n-1} s^{\prime \prime}+D_{n} s^{n-2}\left[s^{\prime}\right]^{2}\right\}
\end{aligned}
$$

The expansion has this form because the first time derivative is applied to a fraction whose expansion contains norder terms of the form $s^{n-1} s^{\prime}$ and $s^{n}$. After the derivation we obtain n-order terms of the form $s^{n-1} s^{\prime \prime}, s^{n-2}\left[s^{\prime}\right]^{2}$ and $s^{n-1} s^{\prime} . s^{\prime}$ and $s^{\prime \prime}$ are first and second time derivatives of $s$. In addition we still have n-order terms of the generic form $s^{n}$ and $s^{\prime} s^{n-1}$ coming from the expansion of the right hand side of (13). Hence the general n-order term is $A_{n} s^{n}+B_{n} s^{n-1} s^{\prime}+C_{n} s^{n-1} s^{\prime \prime}+D_{n} s^{n-2}\left[s^{\prime}\right]^{2}$ where all the parameters $A_{n}, B_{n}, C_{n}, D_{n}$ depend on the laser parameters.

\subsection{Method of Harmonic Input for obtaining Volterra transfer functions $H_{n}$}

The Harmonic Input Method (HIM) allows us to find directly all the $H_{n}$ 's as described previously. The different $H_{n}$ 's are found by direct identification of the $G_{k l m \ldots}$ coefficients. For instance, we have:

$$
G_{000}=0, G_{100}=H_{1}\left(j \omega_{1}\right), G_{110}=H_{2}\left(j \omega_{1}, j \omega_{2}\right), \ldots
$$

\subsection{Order by order distortions}

Starting from the two laser rate equations, Darcie et al. [6] derived formulae pertaining to the second order, third order and intermodulation distortions for a channel excited by a superposition of two signals with angular frequencies 
$\omega_{1}$ and $\omega_{2}$. The second and third order distortions are calculated respectively at $2 \omega_{1}$ and $3 \omega_{1}$ whereas the intermodulation distortions are evaluated at $2 \omega_{1}-\omega_{2}$ and $2 \omega_{2}-\omega_{1}$. From formula (7), we can write these distortions as: a- Second order:

$$
M_{2}\left(j \omega_{1}, j \omega_{1}\right)=20 \log _{10}\left\{\frac{\left|H_{2}\left(j \omega_{1}, j \omega_{1}\right)\right|}{2\left|H_{1}\left(j \omega_{1}\right) H_{1}\left(j \omega_{1}\right)\right|}\right\},
$$

b- Third order:

$$
\begin{aligned}
& M_{3}\left(j \omega_{1}, j \omega_{1}, j \omega_{1}\right)= \\
& 20 \log _{10}\left\{\frac{\left|H_{3}\left(j \omega_{1}, j \omega_{1}, j \omega_{1}\right)\right|}{4\left|H_{1}\left(j \omega_{1}\right) H_{1}\left(j \omega_{1}\right) H_{1}\left(j \omega_{1}\right)\right|}\right\}
\end{aligned}
$$

c- Intermodulation:

$$
\begin{aligned}
& M_{3}\left(j \omega_{1}, j \omega_{1},-j \omega_{2}\right)= \\
& 20 \log _{10}\left\{\frac{\left|H_{3}\left(j \omega_{1}, j \omega_{1},-j \omega_{2}\right)\right|}{4\left|H_{1}\left(j \omega_{1}\right) H_{1}\left(j \omega_{1}\right) H_{1}\left(-j \omega_{2}\right)\right|}\right\} \\
& M_{3}\left(j \omega_{2}, j \omega_{2},-j \omega_{1}\right)= \\
& 20 \log _{10}\left\{\frac{\left|H_{3}\left(j \omega_{2}, j \omega_{2},-j \omega_{1}\right)\right|}{4\left|H_{1}\left(j \omega_{2}\right) H_{1}\left(j \omega_{2}\right) H_{1}\left(-j \omega_{1}\right)\right|}\right\}
\end{aligned}
$$

The HIM allows us to calculate the values of the various $H_{n}$ 's for $\mathrm{n}=1,2$ and 3 . As an illustration of the procedure, we calculate $H_{1}$ and $H_{2}$ after performing steps 4.1 and 4.2 and having obtained the expansion of (13) to the specified order $[12,13]$. First, we calculate $H_{1}$ after simply using $i=\exp (j \omega t)$ in (14) and:

$$
s=\sum_{k=0}^{\infty} G_{k} \exp [j(k \omega) t]
$$

The term $G_{1}$ [with the identification of the terms multiplying $\exp (j \omega t)]$ obeys the relation:

$$
1=\left[A_{1}+j \omega B_{1}-\omega^{2} C_{1}\right] G_{1}
$$

where $A_{1}, B_{1}$ and $C_{1}$ depend on the laser parameters. Using the HIM, we can write:

$$
H_{1}(j \omega)=G_{1}=1 /\left[A_{1}+j \omega B_{1}-\omega^{2} C_{1}\right]
$$

The modulus of $H_{1}(j \omega)$ equal to $1 / \sqrt{\left(A_{1}-\omega^{2} C_{1}\right)^{2}+\left(\omega B_{1}\right)^{2}}$ is what Darcie et al. [6] call the small-signal frequency response $R(\omega)$. In order to calculate second order terms, we use $i(t)=\exp \left(j \omega_{1} t\right)+\epsilon x p\left(j \omega_{2} t\right)$ in equation (14) [truncated to second order] along with:

$$
s=\sum_{k, l=0}^{\infty} G_{k l} \exp \left[j\left(k \omega_{1}+l \omega_{2}\right) t\right]
$$

The various $G_{k l}$ are obtained from the following [12, 13]: $G_{00}=0 ., G_{10}=H_{1}\left(j \omega_{1}\right), G_{01}=H_{1}\left(j \omega_{2}\right)$ and $G_{11}=$
$H_{2}\left(j \omega_{1}, j \omega_{2}\right)$. Identification of the terms multiplying $\exp \left(j\left[\omega_{1}+\omega_{2}\right] t\right)$ yields:

$$
\begin{aligned}
& 0=\left[A_{1}+j\left(\omega_{1}+\omega_{2}\right) B_{1}-\left(\omega_{1}+\omega_{2}\right)^{2} C_{1}\right] G_{11} \\
& +\left[A_{2} G_{01} G_{10}+A_{2} G_{10} G_{01}+j \omega_{1} B_{2} G_{01} G_{10}\right. \\
& +j \omega_{2} B_{2} G_{10} G_{01}-\omega_{1}^{2} C_{2} G_{01} G_{10}-\omega_{2}^{2} C_{2} G_{10} G_{01} \\
& \left.-\omega_{1} \omega_{2} D_{2} G_{01} G_{10}-\omega_{1} \omega_{2} D_{2} G_{10} G_{01}\right]
\end{aligned}
$$

With the use of (22) this can be written as:

$$
\begin{aligned}
& H_{2}\left(j \omega_{1}, j \omega_{2}\right)=G_{11}=-\left[2 A_{2}+j\left(\omega_{1}+\omega_{2}\right) B_{2}\right. \\
& \left.\quad-\left(\omega_{1}^{2}+\omega_{2}^{2}\right) C_{2}-2 \omega_{1} \omega_{2} D_{2}\right] \\
& \times H_{1}\left(j \omega_{1}\right) H_{1}\left(j \omega_{2}\right) H_{1}\left(j\left[\omega_{1}+\omega_{2}\right]\right)
\end{aligned}
$$

The second order distortions are obtained from (16) once the expressions of $A_{2}, B_{2}, C_{2}, D_{2}$ are found from the direct expansion of (13).

\section{Conclusion}

In this work, we outline a general and systematic method to evaluate Harmonic and Intermodulation distortions that occur when a single frequency signal or a superposition of several signals with different frequencies propagate in a non-linear MO write channel at high data rates. The method is generalizable to any order and pertains to cases where a non-trivial relationship between input and output exists at the LD, LM or MD level of the channel.

Earlier studies [7] dealt with second-harmonic generation, optical rectification and other non-linear effects at a single frequency whereas our work is applicable to several input and output frequencies paving the way to the study of modulation and multiplexing in the non-linear MO write channel.

\section{References}

1) S. Hwang, K. S., and H. Choi, IEICE E84-B, 614 (2001).

2) J. Lubbock and S. Bansal, Proc. of the IEE 116, 2075 (1969).

3) J. J. Bussgang, L. Ehrman, and J. W. Graham, Proc. of the IEEE 62, 1088 (1974).

4) M. Toner and G. W. Roberts, IEEE Trans. on Circuits and Systems CAS II-43, 608 (1996).

5) C. Wilker and C. F. Carter, Proceedings of the 50-th Automatic RF Techniques Group Conference ARFTG Fall, 1 (1997).

6) T. Darcie, R. Tucker, and G. Sullivan, Electronics Letters 22, 619 (1986).

7) S. Lim, J. Osman, and D. Tilley, J. Phys. D 32, 755 (1999).

8) R. Monaco, A. Andreone, and F. Palomba, J. App. Phys. 88, $2898(2000)$

9) C. Tannous, A. Fessant, J. Gieraltowski, J. Jay, D. Spenato, J. Langlois, and H. Le Gall, Journal de Physique IV 8, 331 (1998).

10) E. Van de Riet and F. Roozeboom, J. App. Phys. 81, 350 (1997).

11) H. Le Gall, Journal de Physique C1-32, 590 (1971).

12) E. Bedrosian and S. O. Rice, Proc. of the IEEE 59, 1688 (1971).

13) A. Mircea and H. Sinnreich, Proc. of the IEE 116, 1644 (1969).

Received March 11, 2002; Accepted June 10, 2002 